\title{
An analytical approach for the EMHD Williamson nanofluid over nonlinear sheet with double stratification and Ohmic dissipation
}

\author{
Muhammad Bilal ${ }^{1}$ and Inam Ul Haq ${ }^{1}$ \\ ${ }^{1}$ The University of Lahore - Gujrat Campus
}

August 12, 2020

\begin{abstract}
This study emphasized the computational aspects of the electromagnetohydrodynamic (EMHD) flow of Williamson nanofluid with variable viscosity and dissipation effects over a nonlinearly expanding sheet. The viscosity of the fluid depends upon temperature and thermal diffusion. Due to nonlinear expansion of sheet, a solutal and thermal stratification phenomenon are also incorporated. A uitable transformation is applied to the basic mathematical problem to convert the system of partial differential equations (PDEs) into nonlinear ordinary differential equations (ODEs). An efficient analytical approach known as HAM (homotopy analysis method) is used to achieve the local similar solutions. The attributes of commanding variables, such as the viscosity parameter, Hartman number, Lewis number, Weissenberg number, Brownian motion parameter, stretching index, and stratification parameters are related to velocity, temperature, and concentration profiles through graphs and tables. Convergence table and h-curves are drawn for the optimal solution through HAM. Numerical values are well tabulated for the study of skin-friction and Sherwood numbers against the different parameters.
\end{abstract}

\section{Hosted file}

manuscript.pdf available at https://authorea.com/users/350498/articles/475321-ananalytical-approach-for-the-emh-williamson-nanofluid-over-nonlinear-sheet-with-doublestratification-and-ohmic-dissipation 

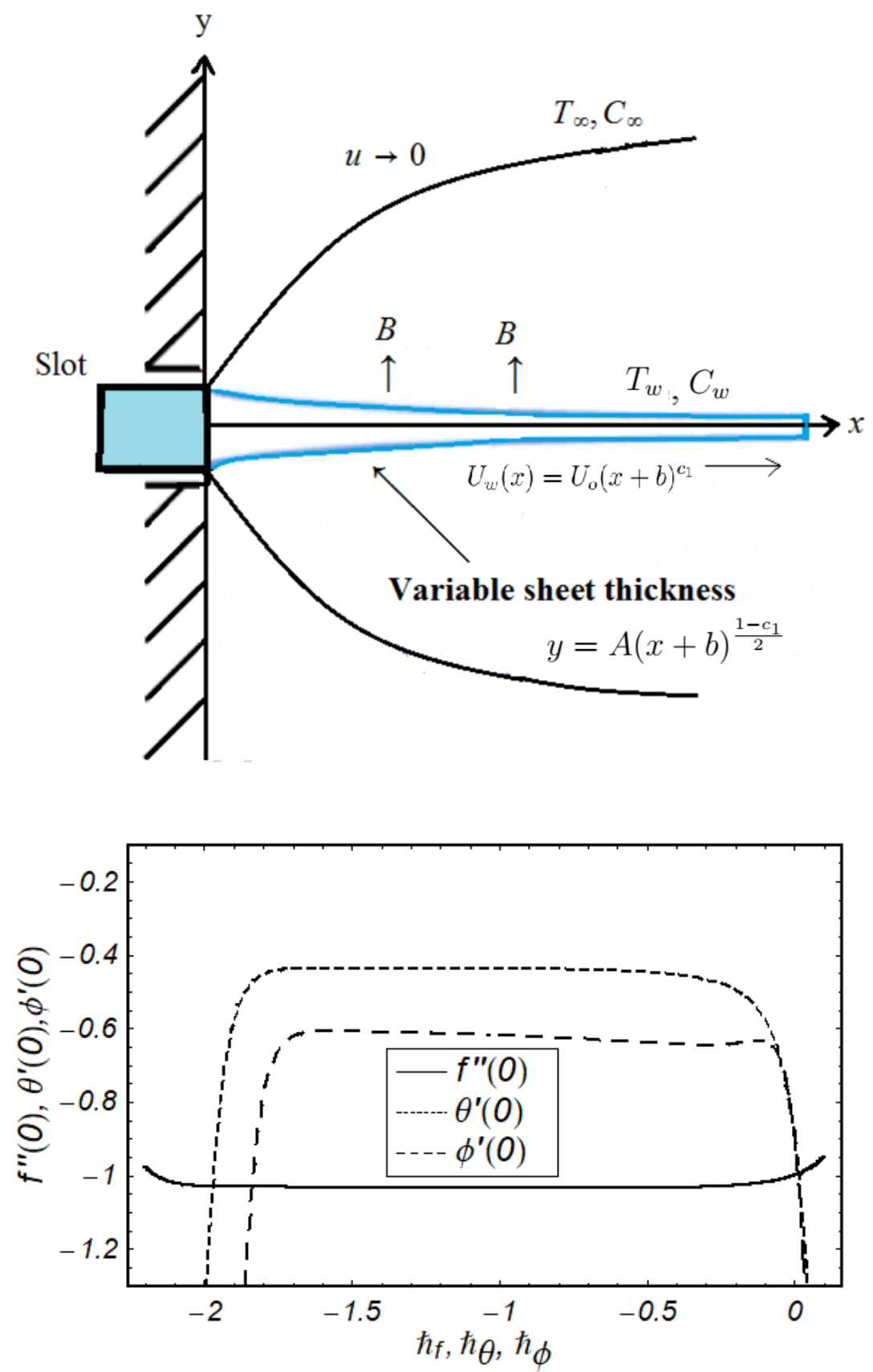
figures/WeF/WeF-eps-converted-to.pdf 
figures $/ k 1 F / k 1 F-e p s-c o n v e r t e d-t o \cdot p d f$ 
figures/c1F/c1F-eps-converted-to.pdf 
figures/E1F/E1F-eps-converted-to.pdf 
figures/alphT/alphT-eps-converted-to.pdf 
figures/c1T/c1T-eps-converted-to.pdf 
figures/E1T/E1T-eps-converted-to.pdf 
figures/EcT/EcT-eps-converted-to.pdf 
figures/NbT/NbT-eps-converted-to.pdf 
figures/PrT/PrT-eps-converted-to.pdf 
figures/StT/StT-eps-converted-to.pdf 
figures/NbP/NbP-eps-converted-to.pdf 
figures/LeP/LeP-eps-converted-to.pdf 
figures/NtP/NtP-eps-converted-to.pdf 
figures/ScP/ScP-eps-converted-to.pdf 
figures/a1F/a1F-eps-converted-to.pdf 